\title{
Rehidratación oral de lactantes con diarrea aguda en servicios de emergencia
}

\author{
Dr. Joxe M. l'garte S.1,3: Dr. Iiduardo Clávez C,2,4*; Dr. Dino Curotto A,1,2; \\ Dra. Gloria Durán S.4: Dr. C'urjos Toro A. ${ }^{1}$
}

\section{Oral rehydration in infants with acute diarrhea}

\begin{abstract}
Ural rchydration has proved an etlective treatment in patient with acute diarrhea. Oral rehydration with whO: solution (ORSi was used in 89 infancs (age 1 to 20 months, nean 3.5 months) with mild to moderate debydration. Group A patients (n $=54$ ) were given $50 \mathrm{~mL} i \mathrm{~kg}$ of ORS when mildly dehydrated and $100 \mathrm{~mL} / \mathrm{kg}$ when this was or moderate degrec, in the first 4 hours, followed by plain water (25 and $50 \mathrm{~mL} / \mathrm{kg}$, respectively) in the next 2 hours. Grotup b patien1s $(n=35)$ were tiven diluted ORS (2/3 5uength) 75 and $150 \mathrm{~mL} / \mathrm{kg}$ for mild and moderate dthydration respectively) along the wlyole 6 ljours period. Nasogastric tubes were used as necssary. At the end of 6 hours body wight increased by $5 \%$ (mean) and hydration state was considered normal by clinical standards in all patients of both groups. Torty percent of patients came back to control on next day, $88 \%$ of them were normally hydrated. Considerable reduction of the number of hospital admissions is possible by the rational use of ORS in pitient $s$ with dcute diarrea and mild or moderate dehydration.

(Key words: acute diarrloca, dehydration, oral rehydration).
\end{abstract}

El sindrome diarreico agudo es causa frecuente de ponsulta en nuestro medio, especialmente en terano. ${ }^{1-3}$

Si bien la mortalidad por esta entidad ha disminutido notablemente ${ }^{4-7}$ gracias al tratamiento oportuno $y$ eficaz de sus complicaciones, el número de hospitalizaciones continúa siendo considerable. ${ }^{a}$ Esta situación no sólo significa costos económicos importantes y daños psicológicos para el niño y sus padres, sino también la exposición en el Hospital a riesgos adicionales que están en último tér mino entre las causas más frecuentes de muerte en niños con diarrea (infección intrahospitalaria; diarrea de evolución prolongada; desnutrición secundaria).

La corrección hidroelectrolítica y ácido-base

1. Depto. de Pediatría y Cirugia Infantil (Central). Facultad de Medicina Universidad de Chile.

2. Servicio de Pediatría. Hospital Paula Jaraquemada.

3. Servicio de Urgencia. Consultorio Maipú.

4. Servicio de Urgencia. Hospital Parroquial de San Bernardo.

* Trabajo de ingreso a la Sociedad Chilena de Pediatría. por la vía parenteral ha sido fundamental en la disminución de la mortalidad por diarrea aguda. Sin embargo significa necesariamente hospitalizar al niño deshidratado. El advenimiento en estas últimas dos décadas del uso de sales de rehidratación oral ha constituido una alternativa ef $i$ $\mathrm{caz}^{9-13}$ cuando son usadas en forma oportuna, con las ventajas de no requerir equipo complicado, ser de bajo costo, fácil preparación y uso, mejorar rápidamente el apetito disminuyendo el riesgo de desnutrición secundaria y ser similar para todos los grupos etarios. ${ }^{14,15}$

En nuestro medio se ha usado con éxito la rehidratación oral en lactantes hospitalizados por diarrea aguda, ${ }^{16} \sin$ embargo, las experiencias a nivel ambulatorio son escasas. $1 \cdot 16$

El propósito de nuestro trabajo consiste en demostrar que es posible disminuir la frecuencia de hospitalización por diarrea aguda complicada del lactante mediante rehidratación oral en los servicios de urgencia (nivel de atención primaria). ${ }^{17}$

\section{MATERIAL Y METODO}

Se aplicaron programas de rehidratación oral en dos servicios de urgencia: posta de Majpú depenđiente del 
Hospital Paula Jaraquemada (grupo A) y posta del Hospital Parroquial de San Bernardo (grupo B), duran te 4 meses de vera to (diciembre 1986 - marzo 1987).

Se admitieton 89 lactantes, $(A ; n=54 ; B: n=35)$ cuyas cdades fuctuaban entre I y 20 meses $(\bar{x}$ A: 8 meses; $\bar{x}$ B: 7 mescs), portadores de diartea aguda con deshidratación. De los pacientes del grupo A, $30(56 \%)$ tenían deshidratación leve y 24 (44\% moderada. En el srupo B las proporciones correspondicntes fueron 29 (82\%) con deșjdratación ltue y 6 (18\% moderada. En total 59 pacientes $(66 \%$ tenian deshidratación leve y 30 (34\%) moderada. Ia magnitud de la deshidratación se craluó sobre bases clínicis 18.19 Durante el período de cstudio sólo fueton hospitalizados para terapia endoverosa los lactantes que al consultar sc cncontsaban gravemente deshidratados (shock hipovolémico) o conían acidosis clínica severa. Se excluyeron del estudio lus desnutridos pluricarcncjales: los desnutridos calórico protejcos grados II $y$ III (sceún tablas NCHSj con deshidratación moderada o grave: los recién nacidos y lactartes en cue la diarrea $※$ presentaba como una expresión mis de una infección generalizada. I: l vomito no fue considerado criterio de cxclusión.

Los niños ingresaron son su madre o acompantante qquićn, en algunos casos (20\%), adistinistró la solución medjante cucharas, dependiendo de la aceptación del nùn, la disposición de la madre y personal $\rfloor$ cooperar. In los demás niños la solución se dió por uastroclisis. De todos modos la madre acompanó al niño durante todo el procedimiento. Todos los niños lueron pesados antex de iniciar su rchidrataxión.

En ambos grupos se emplé́ la solución oral OMS: INICEF ( $\mathrm{Na}^{+} 90 \mathrm{mLq} / \mathrm{L} ; \mathrm{K}^{+} 20 \mathrm{mEq} / \mathrm{L} ; \mathrm{HCO}_{3}{ }^{-3} 30$ $\mathrm{mEq} / \mathrm{L}:$ glucosa $20 \mathrm{~g} / \mathrm{L}=111 \mathrm{~m} \mathrm{Mol} / \mathrm{L})$. Fn el grupo A se empleó sin diluir en las primeras 4 horas de tratamicnto (dishidratacion leve $50 \mathrm{~mL} / \mathrm{kg}$, moderada 100 $\mathrm{mL} / \mathrm{kg}$ ), siendo remplazada por agua pura para las 2 horas siguientes (deshjdratación leve $25 \mathrm{~mL} / \mathrm{kg}$, mode. rado $50 \mathrm{~mL} / \mathrm{kg}$ ). Cn el grupo $B$ se emplcó la solución diluida a razón de 2 partes por 1 de agua pura suministrándola a lo largo de todo el período inicial de 6 horas. De esta manera los volúmenes totales aportados durante las 6 horak en ambos grupos fueron de $75 \mathrm{~mL} / \mathrm{kg}$ de peso en casos de deshidratación leve y $150 \mathrm{~mL} / \mathrm{kg}$ a los ninos con deshidratación moderada.

Para la gastroclisis se emplearon matraves $y$ equipos plásticos de perfusión usados, lavados con agua porable.
En los casos en que la solución fue administrada por la madre, se le cntregó preparada on envases de vidrios previamente esterilizados. F, cumplimiento de la indicación fue controlado a intervalos de 2 horas y alcanzó $100 \%$ para ambos grupos. Al cabo de las $6 \mathrm{~h}$ se pesaron nuevamente los niños, consignando el estado de hidratación y de conciencia. Proviamente se labía establecido que el procedimiento debía suspenderse si aparecían o jersist ian vónitos o sc agregaba distensión abdominalo ambos, durante la observación. Cualquier niño cuya deshidratación persistiese o empeorase después de las 6 horas de hidratación oral, seria hospitalizado. En caso de mejoría de los nin̄os continuarian su tratamiento en vatsa con control 24 horas después del egreso en el mismo servicio de urgencia.

Lil tratamiento indicado a las madres para la casa (mantención de la hidratación y nutrición) consistió para el grupo $A \mathrm{cn}$ solución OMS (Na 90$) 100 \mathrm{~mL} / \mathrm{kg} \mathrm{x}$ 24 l y para el grupo $B$, al mismo volumen en agua de canela o aguis de arroz.

Para ambos grupos la indicación de alimentos consistió en leclee materna según demanda o una fórmula láctea constituida por leche de vaca en polvo $5 \mathrm{~g} \%$ azúcar $5 \mathrm{~g} \%$ y crema de arroz $5 \mathrm{~g} \%$ No se emplearon antibióticos ni anticolinéreicos.

Para la cvaluación de las 24 horas se emplearon criterios semejantes que d las 6 horas: se dió el alta a los fuctantes hidratados indicándoseles control en su consultorie 48 horas después; se hospitalizaron los niños que tenían deshidratación moderada insistićndose en la rehjdratación oral por otras 6 horas en los casos con deshidrazación leve (figura 1).

\section{RESULTADOS}

Todos los lactantes de ambos grupos estaban bien hidratados al cabo de 6 horas. Esta apreciación clínica se pudo objetivar a través del incre. mento promed o de peso corporal de $5 \%: 5,6 \%$ (rango 2,1 a 10,7) èt el grupo A y 4,2\% (rango 0,5 a 13,7 ) en el grupo $B$.

Ningún niño presentó vómitos, distensión abdominal importante, o requirió ser hospitalizado en esta etapa.

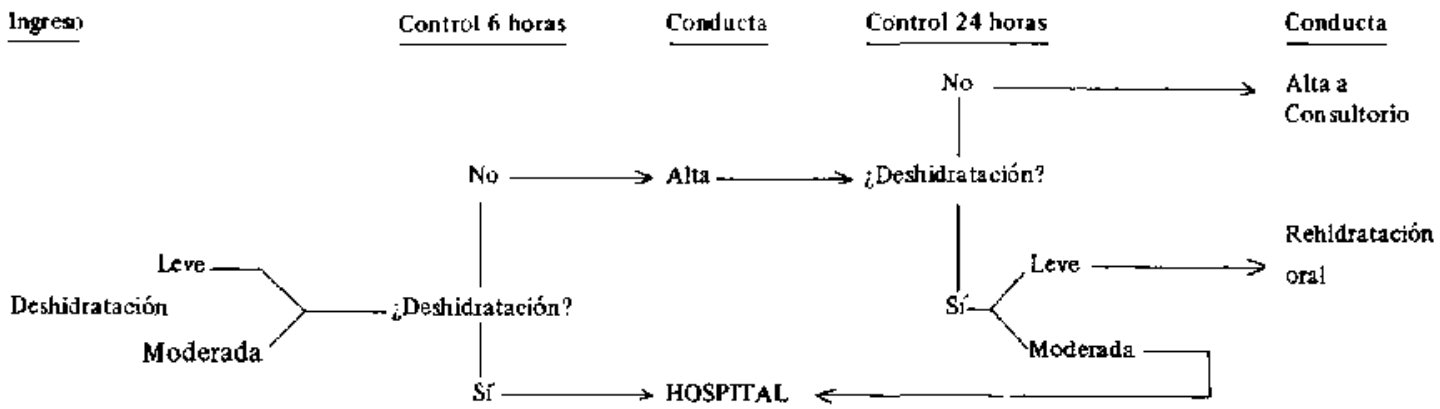

Figura 1: Flujograma de seguimiento y control de lactantes con diarrea aguda y deshidratación leve o moderada tratados con rehidratación oral. 
La asistencia a control 24 horas después del alta fue sólo de $39 \%$. Los 24 riños controlados del grupo A estaban bien hidratados y fueron dados de alta para su tratamiento ambulatorio en consultorio. Sin embargo, en el grupo B, 4 de 11 controlados se labian deshidratado nuevamente $(36,4 \%)$ : en uno, con deshidratación leve, se repitió el tratamiento oral en el mismo servicio de urgencia, esta vez con buen resultado definitivo. Los otros 3 niños requirieron terapia endovenosa en el hospital por deshudratación moderada.

No asistieron al control de 24 horas 54 de 89 nínos ( $61 \%$ ), cuyo seguimiento domiciliario no fue posible.

\section{DISCUSION}

Sin duda la terapia de rehidratación oral fue beneficiosa para este grupo de niños tratados en ambos grupos de urgencia. Todos ellos hubiesen sido hospitalizados segun los criterios vigentes al comienzo de este trabajo, con los consiguientes costos y riesgos. Sin embargo registramos sólo 3 lracasos en 89 experiencias.

Aun si se incluyesen entre los fracasos a todos los pacientes que no concurrieron a control 24 horas después del alta, el tratamiento parenteral pudo evitarse en 32 de los lactantes oon deshidra. tación (36\%). Dado que este estudio se realizó con poblaciones semirurales distantes de otros centros asistenciales, es poco probable que las inasistencias se debiesen de manera predominante al fracaso del tratamiento. Otros estudios han demostrado que ella se debe más bien a la mejoría de los pacientes. (Delucchi MA., Guiraldes E. y cols., observaciones no publicadas).

La aparición de pacientes con de shidratación en el control de 24 horas en el grupo B probablemente se relaciona con el hecho de no habers: indicado soluciones electrolíticas para la etapa de mantención de la hidratación que siguc a la de recuperación inicial.

\section{RESUMEN}

La rehidratación ora! en diarred aguda se considera un método efectivo y seguro en el mundo. No hay un plan nacional para su uso en el nivel primario. Se utilizó rehidratación oral con solución OMS en 89 lactantes entre 1 y 20 meses $(\bar{x}=3,5 \mathrm{~m})$, con deshidratación leve a moderada, admitidos por diarrea aguda a servicio de urgencia de áreas sub-urbanas de Santiago. En el grupo $1(\mathrm{n}=54)$ se administró solución OMS por 4 horas $(50 \mathrm{~mL} / \mathrm{kg}$ de peso cuando la deshidratación era leve y 100 , cuando era moderada), seguida de agua pura por 2 horas $(25$ y $50 \mathrm{~mL} / \mathrm{kg}$ respectivamente). En el grupo $2(n=35)$ se dió por 6 horas solución OMS diluida $2 / 3$ en volúmenes de 75 y $150 \mathrm{~mL} / \mathrm{kg}$ de peso, en la deshidratación leve y moderada respectivamente. Cuando fue necesario, se emplearon sondas nasogástricas. Al cabo de 6 horas de tratamiento, el incremento de peso corporal promedio fue de $5,0 \%$ y todos estaban bien hidratados en ambos grupos. Fueron enviados a su domicilio con indicaciones de dimentación e hidratación. Al dia siguiente, concurrió a control 39,3\% de los pacientes. De ellos, $88,6 \%$ estaban bien hidratados. Se demuestra que puede disminuirse, considerablemente, el número de hospitalizaciones por diarrea aguda con un programa coherente de hidratación oral en servi. cios de urgencia.

\section{REFERENCIAS}

1. Araya M., Figteroa A., Espinoza J., Montecinos $V$. Spencer $E_{1}$, Brunser $O$ : Acute diarthoeal disease in children under $?$ years of age a peri-urban slum of Santiano, Chile. J Hyg Camb 1985; 95: 457-467.

2. Aray'a $M$. Figueroa $G$., Espinoza $J$, Zarter $X$. Bruiser $O .:$ Acute diarrhoea and asymptomatic infection in Clilean prescloolers of low and high socio-economic strata. Acta Pediatr Scand 1986; 75 : $645 \div 651$.

3. Cicn I.C.: Interactions of diarrhea and malnutrition. In: Chen L.C., Schrimshaw N.S., eds. Diarrhea and Maluutrition: Interaction, mechanisms and interventions. 1 st cd. New York: Plenum Press, 1983: 3-19.

4. Nore Health Organization: Levels, trends, differentials and causes of intant and early childhood mortality in Latin America. World Health Statistics Quarterly 1982; $32: 147$.

5. Ministerio de Salud Publica: Departamento de Planificación (1983). Definiciones y causas de muerte. Anvario. Santiago, Chile.

6. Nonckeberg F. and Riumalló J.: (1983). Nutritional recovery centers: the Chilean experience. In Nutrition Intervention: strategies in National Develirpment. Ed. B.A. Underwood, pp. 189-199. New York: Academic Press.

7. Brumser O. Figueroa (j., Aroya M. and Espinoza $J$.: (1984). Infections and diarrheal disease. In Malnutrition Determinants and Consequenses. Ed., P.L. White and N. Setvery. In Current Topics in Nutrition and Diseases 10, p 59. New York: Alan R. Liss. 
8. Tieffenberg I.A., Segure T.; Romero M.l. and Brunser O.: (1983). Clinical diagnosis in chidren under 5 years of age who attend a periurban Health Center in Santiago, Chile. Relations to acute diarrhea. XXI st Annual Meeting of Latin American Society for Pediatric Research, Montcvideo, Uruguay, Abstract 61.

9. Hirschhorn N., Mc Carthy B.J., Ranney B.. Hirschhorn A., Woodward N.E.: Ad libitum oral glucose-clectrolyte therapy for acute diarhea in Apache children. J Pediatr 1973; 83: 562-571.

10. Pizarro D., Posado (i, Levine M. Mohs E.: Oral rehydration of infants with acute diarrohoeal dehydration. A practical method. I Trop Med Hyg 1980;83: 241-245.

11. Pizaro D., Poszda G., Nalin D. Mata L., Mohs E.: Rehidratución por vía oral y su mantenimiento en pacientes de 0 a 3 meses de edad deshidratados por diarrea. Bol Med Hosp Intant Mex 1980; 37: 879.8B2.

12. Tavior P.R., Mcrson M.H., Black R.E., Wizanur Rahman. A.S.M., Yunus M.D., Shim A.R.M.A., Yolken R.H.: Oral rehydration therapy for treatment of rotavirus diarrltoea in a eural treatment centre in Bangladesll, Arc] Dis Child 1980:55: 376-381.
13. Booth I.W., Levine M.M., Harries J.T.: Oral rehydration therapy in acutc diarrea in childhood. J Pediatr Gastroenterol Nutr 1984; 3: 491-499.

14. Vasundhara K. Tolia, M.B.B.S. and Reuben $S$. Dubois M.B.B.S. F.R.A.C.P.: Uptate on Oral Rehydration: Its place in treatment of Acute Gastroenteritis. Pediatr Ann 1985: 14: 295-303.

15. Hirschborn $N$.: The treatment of acute diarrhes in children - A historical and physiological perspective. Am J Clin Nutr 1980: 33: 637-663.

16. Duffau G., Emilfork M., Culderón A.: Evaluación de dos fórmulas para hidratación oral en el síndrome diarré́co agudo con deshidratación del lactante. Bol Med Hosp Infant Mex 1982; 39: 729-736.

17. Pizarro D., Posadn G., Mohs E., Levine M., Nalint D.: Evaluation of oral therapy for infant diarrohoca in an emergency room setting: the acute episode as an opportunity for instructing mothers in home treatment. Bult WHO 1979; 5 ?: 983-986.

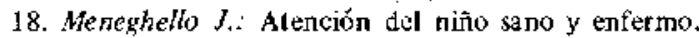
Santiago de Chile: Editorial Andrés Bello, 1978; 1040-1065.

19. Duffau $G$.: Síndrome diarreico agudo del lactante. Santiago de Chíle: Editorial Andrés Bello, 1978: 53-61. 